\title{
Event-Triggered Faults Tolerant Control for Stochastic Systems with Time Delays
}

\author{
Ling Huang, Xuhuan Xie, and Wenbo Xie \\ School of Automation, Harbin University of Science and Technology, Harbin 150080, China \\ Correspondence should be addressed to Ling Huang; mail_huangling@163.com
}

Received 16 March 2016; Accepted 19 May 2016

Academic Editor: Guo Chen

Copyright (C) 2016 Ling Huang et al. This is an open access article distributed under the Creative Commons Attribution License, which permits unrestricted use, distribution, and reproduction in any medium, provided the original work is properly cited.

\begin{abstract}
This paper is concerned with the state-feedback controller design for stochastic networked control systems (NCSs) with random actuator failures and transmission delays. Firstly, an event-triggered scheme is introduced to optimize the performance of the stochastic NCSs. Secondly, stochastic NCSs under event-triggered scheme are modeled as stochastic time-delay systems. Thirdly, some less conservative delay-dependent stability criteria in terms of linear matrix inequalities for the codesign of both the controller gain and the trigger parameters are obtained by using delay-decomposition technique and convex combination approach. Finally, a numerical example is provided to show the less sampled data transmission and less conservatism of the proposed theory.
\end{abstract}

\section{Introduction}

Networked control systems (NCSs) are control systems wherein the control loops are closed through a certain digital communication network, which have been widely used in many fields, for example, vehicle industry, robot, and unmanned aerial vehicles, because of their benefits, for example, low installation and maintenance costs, high reliability, increased system flexibility, and decreased wiring. However, inserting a network to a control loop also brings a series of network-induced constraints, for example, time delays, packet losses, and competition of multiple nodes accessing network. The network-induced constraints will deteriorate the systems performance or even destabilize the system [1, 2]. Therefore, NCSs with network-induced constraints have gained considerable research interests and numerous results have been published [3-21].

So far, most of the analysis and synthesis for NCSs utilize time-triggered communications scheme, for example, stabilization $[3,4]$, filtering $[5,6]$, and tracking control [7]. It is well known that network-induced constraints are mainly caused by the limited network bandwidth. Moreover, it is very significant to mitigate the networked load to increase the lifespan of the battery of the nodes in wireless NCSs [8]. Therefore, to reduce the unnecessary waste of computation and transmitting sample data not only has theoretical importance but also has practical significance. In general, NCSs using event-triggered communication scheme can considerably reduce the network resource occupancy while maintaining the control performance compared to NCSs using time-triggered communication scheme [9]. So, event-triggered control for NCSs has received considerable attention in the past decade because of energy conservation and optimal performance, especially in the wireless NCSs [8], for example, event-triggered state-feedback control [10, 11], event-triggered guaranteed cost control [12], event-triggered dynamic output feedback control [13], event-triggered output tracking control [14], and event-triggered fuzzy filtering [15]. Considering the event-triggered control for NCS with stochastic perturbations [22-25], limited work has been reported in the open literatures. In [16], event-triggered stabilization for networked stochastic systems with multiplicative noise and network-induced delays has been investigated. However, the time delays in state and the effect of fault are not taken into account. On the one hand, fault tolerant control for NCSs has attracted great attention over the past decades because actuator faults caused by actuator aging or actuator in a hostile environment will have a great threat to the security and the reliability of NCSs [17-21]. On the other hand, systems with delays are ubiquitous, for example, 
NCSs, biology systems, and hydraulic rolling mill systems $[2,26-28]$. To the best of the authors' knowledge, there is no result reported in the open literatures on event-triggered faults tolerant control for stochastic systems with time delays. This motivates the study presented in the paper.

In the paper, we deal with the state-feedback controller design for stochastic NCSs with random actuator failures and transmission delays. The main works of this paper are as follows: (1) a triggered scheme is introduced to optimize the performance of stochastic NCSs; (2) stochastic NCSs under an event-triggered scheme are modeled as stochastic timedelays systems; (3) some less conservative stability criteria for the codesign of both the controller gain and the trigger parameters are obtained by using delay-decomposition technique and convex combination approach; (4) a practice example on event-triggered control for an unstable batch reactor is provided to show the merits of the proposed theory.

Notation. The notation used throughout the paper is fairly standard. $N^{T}$ and $N^{-1}$ denote the transpose and the inverse of matrix $N$, respectively. For a square matrix $M, \operatorname{Sym}\{M\}$ is defined as $M+M^{T}$. The symmetric term in a matrix is denoted by $*$; for example, $\left[\begin{array}{ll}X & Y \\ * & Z\end{array}\right]=\left[\begin{array}{cc}X & Y \\ Y^{T} & Z\end{array}\right]$. The notation $P=P^{T}>0$ $\left(P=P^{T} \geq 0\right)$ means that the matrix $P$ is real symmetric positive definite (positive semidefinite). $\left(\Omega, F,\left\{F_{t}\right\}_{t \geq 0}, \mathbb{P}\right)$ is a complete probability space and $E\{\cdot\}$ stands for the expectation operator with respect to the probability measure. $\operatorname{diag}\{\cdots\}$ stands for a block diagonal matrix. Matrices, if not explicitly stated, are assumed to have appropriate dimensions.

\section{Problem Formulation}

Consider a linear system with state delay and Itô process, which is represented by the following stochastic differential equation:

$$
\begin{aligned}
d x(t)= & {\left[A x(t)+A_{d} x\left(t-\tau_{d}\right)+B u(t)\right] d t } \\
& +\left[H x(t)+H_{d} x\left(t-\tau_{d}\right)\right] d \omega(t), \quad t>0, \\
x(t)= & \varphi(t), \quad t \in\left[-\tau_{d}, 0\right],
\end{aligned}
$$

where $x(t) \in \mathbb{R}^{n}$ and $u(t) \in \mathbb{R}^{m}$ denote the state vector and the control input of the system, respectively, $\tau_{d}$ is a constant delay, $A, A_{d}, B, H$, and $H_{d}$ are some constant matrices with appropriate dimensions, $\omega(t)$ is a scalar standard Brownian Motion defined on a complete probability space $\left(\Omega, F,\left\{F_{t}\right\}_{t \geq 0}, \mathbb{P}\right)$ with a natural filtration $\left\{F_{t}\right\}_{t \geq 0}$ and satisfies $E\{d \omega(t)\}=0$ and $E\left\{d \omega(t)^{2}\right\}=d t$, and $\varphi(t)$ is a continuous vector valued initial function defined on $\left[-\tau_{d}, 0\right]$.

The stochastic system controlled by a network is described in Figure 1.

To simplify the exposition, we make the following assumptions.

Assumption 1. All the system states variables of the controlled plant are available for a state-feedback control. The sensor is time-triggered with a constant sampling period $h$. The

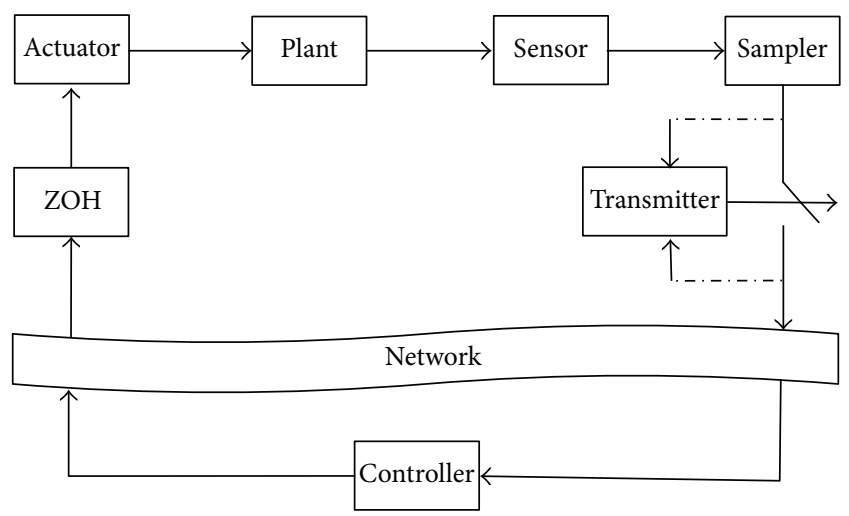

FIgURE 1: A framework of stochastic NCSs under an event-triggered communication scheme.

sampling sequence is described by the set $x(j h), j=1,2$, $\ldots, \infty$.

Assumption 2. The sampled data is transmitted in a single packet, and the packet losses do not occur in transmission. Whether the packets should be transmitted or not over network is determined by an event-triggered communication scheme. The successfully transmitted packet sequence is described by the set $x\left(t_{k} h\right), k \in\{1,2, \ldots, \infty\}$.

Assumption 3. The controllers and the actuators are eventtriggered. The control input at the actuator is generated by a zero-order-holder $(\mathrm{ZOH})$ with the holding time $t \in\left[t_{k} h+\right.$ $\tau_{t_{k}}, t_{k+1} h+\tau_{t_{k+1}}$, where $\tau_{t_{k}}$ is the communication delay. Before the first control signal reaches the plant, the control input $u(t)=0$.

The following state-feedback control law is employed for system (1):

$$
u(t)=K x(t)
$$

Considering the effect of the transmission delay, (2) can be rewritten as

$$
u(t)=K x\left(t_{k} h\right), \quad t \in\left[t_{k} h+\tau_{t_{k}}, t_{k+1} h+\tau_{t_{k+1}}\right] .
$$

In order to improve the performance of the NCSs, the following event-triggered communication scheme is introduced to reduce the energy cost and the adverse effect of the transmission delay on performance of the NCSs:

$$
\begin{aligned}
& {\left[x\left(t_{k} h\right)-x\left(t_{k} h+l h\right)\right]^{T} \Omega_{2}\left[x\left(t_{k} h\right)-x\left(t_{k} h+l h\right)\right]} \\
& \quad \geq \delta x^{T}\left(t_{k} h\right) \Omega_{1} x\left(t_{k} h\right),
\end{aligned}
$$

where $l=0,1, \ldots, t_{k+1}-t_{k}-1,0 \leq \delta<1$, and $\Omega_{1}$ and $\Omega_{2}$ are the positive definite weighting matrices to be designed.

Remark 4. Only the sampled data satisfying inequality (4) will be transmitted since the transmitter in Figure 1 has a logic function to determine whether one should be transmitted or not; when $\delta=0$, inequality (4) holds for all the sampled 
state; hence, it shrinks to the periodic release case in $[17,18]$. Obviously, the amount of transferred data can be reduced by set $\delta \neq 0$ in (4). If the amount of transferred data is reduced, the networked load will be reduced. Then, the energy cost used to transmit the unnecessary sampled data to the controller will be saved, and the adverse effect of the transmission delay on performance of the NCSs will be reduced since the transmission delay is mainly caused by the network traffic congestion.

Remark 5. A similar step to the one used in [16], two different weighting matrices $\Omega_{1}$ and $\Omega_{2}$ are introduced in event-triggering communication scheme (4) to obtain less conservative results. We further illustrate this in the following numerical example.

For a detailed timing analysis, we divide the holding interval of ZOH $t \in\left[t_{k} h+\tau_{t_{k}}, t_{k+1} h+\tau_{t_{k+1}}\right]$ into the following subsets:

$$
\begin{aligned}
& {\left[t_{k} h+l h+\tau_{t_{k}}, t_{k} h+(l+1) h+\tau_{t_{k}}\right]} \\
& \cup\left[t_{k+1} h-h+\tau_{t_{k}}, t_{k+1} h+\tau_{t_{k+1}}\right] \\
& \quad l=0,1, \ldots, t_{k+1}-t_{k}-2 .
\end{aligned}
$$

Define the following new variables:

$$
\begin{aligned}
& \tau(t)=t-t_{k} h-l h, \\
& e(t)=x\left(t_{k} h\right)-x\left(t_{k} h+l h\right),
\end{aligned}
$$

$$
l=0,1, \ldots, t_{k+1}-t_{k}-1
$$

Then, we have

$$
0<\tau_{1} \leq \tau(t) \leq h+\bar{\tau} \triangleq \tau_{2} .
$$

The event-triggered algorithm (4) can be rewritten as

$$
\begin{aligned}
& e^{T}(t) \Omega_{2} e(t) \\
& \quad \geq \delta(e(t)+x(t-\tau(t)))^{T} \Omega_{1}(e(t)+x(t-\tau(t))) .
\end{aligned}
$$

Considering the effect of the stochastic actuator failure, the relationship between the control input $u(t)$ and the real actuator output $\widehat{u}(t)$ is described as follows:

$$
\widehat{u}(t)=\Lambda u(t),
$$

where

$$
\Lambda=\operatorname{diag}\left\{\Lambda_{1}, \Lambda_{2}, \ldots, \Lambda_{m}\right\}, \quad \Lambda_{i} \in[0, \theta],
$$

where $\theta \geq 1$ and $\Lambda_{i}$ are the random variables which describe the relationship between the real executive amount of $i$ th actuator and the control input for ith actuator. $\Lambda_{i}=0$ represents that the $i$ th actuator is completely fails, $\Lambda_{i}=1$ represents that the $i$ th actuator works normally, $0<\Lambda_{i}<$ 1 and $\Lambda_{i}>1$ represent that the real actuator output is smaller and greater than the control input, respectively. The expectation and variance of $\Lambda_{i}$ are $\bar{\Lambda}_{i}$ and $\beta_{i}^{2}$, respectively.

$$
\begin{aligned}
\text { Define } e_{i} & =\operatorname{diag}\{\underbrace{0, \ldots, 0}_{i-1}, I, \underbrace{0, \ldots, 0}_{m-i}\} . \text { Then } \\
\bar{\Lambda} & =\operatorname{diag}\left\{\bar{\Lambda}_{1}, \bar{\Lambda}_{2}, \ldots, \bar{\Lambda}_{m}\right\}=\sum_{i=1}^{m} \bar{\Lambda}_{i} e_{i}, \\
\beta^{2} & =\operatorname{diag}\left\{\beta_{1}^{2}, \beta_{2}^{2}, \ldots, \beta_{m}^{2}\right\}=\sum_{i=1}^{m} \beta_{i}^{2} e_{i} .
\end{aligned}
$$

Based on the above analysis, (3) can be rewritten as

$$
u(t)=B \Lambda K(e(t)+x(t-\tau(t))) .
$$

Substituting (12) into (1), the resultant closed-loop stochastic system with time delays can be expressed as follows:

$$
\begin{aligned}
& d x(t)=\left[A x(t)+A_{d} x\left(t-\tau_{d}\right)+B \Lambda K x(t-\tau(t))\right. \\
& \quad+B \Lambda K e(t)] d t+[H x(t) \\
& \left.\quad+H_{d} x\left(t-\tau_{d}\right)\right] d \omega(t), \quad t>0, \\
& x(t)=\varphi(t), \quad \forall t \in\left[-\max \left\{\tau_{d}, \tau_{2}\right\}, 0\right] .
\end{aligned}
$$

\section{Main Results}

This paper aims to develop a novel state-feedback controller for event-triggered stochastic NCSs with state delay, networked induce delay, and stochastic actuator failure. Before presenting the sufficient stabilization condition, we introduce the following lemmas, which are indispensable for the proofs in the sequel.

Lemma 6 (see [29]). $M_{1}, M_{2}$, and $M_{3}$ are constant matrices with appropriate dimensions; then, a sufficient and necessary condition for

$$
\begin{aligned}
\left(\tau(t)-\tau_{1}\right) M_{1}+\left(\tau_{2}-\tau(t)\right) M_{2}+M_{3} & <0, \\
& \forall \tau(t) \in\left[\tau_{1}, \tau_{2}\right]
\end{aligned}
$$

is that the following inequalities hold simultaneously:

$$
\begin{aligned}
& \left(\tau_{2}-\tau_{1}\right) M_{2}+M_{3}<0, \\
& \left(\tau_{2}-\tau_{1}\right) M_{1}+M_{3}<0 .
\end{aligned}
$$

Lemma 7 ((Schur complement) [30]). Given matrices $Q=$ $Q^{T}, R=R^{T}$, and $S$ with appropriate dimension, the inequality

$$
\left[\begin{array}{ll}
Q & S \\
S^{T} & R
\end{array}\right]>0
$$

is equivalent to $R>0, Q-S R^{-1} S^{T}>0$.

By employing delay-fraction technique, linear convex combination approach, and Lyapunov-Krasovskii stability theorem, the following sufficient stabilization condition for stochastic system (13) with time delay satisfying condition (7) is obtained. 
Theorem 8. For given scalars $\tau_{d}, \tau_{1}, \tau_{2}$, and $\delta$ and a positive integer $\lambda$, the closed-loop stochastic system with time delays (13) and time-varying delay satisfying condition (7) is stochastically asymptotically stable if there exist $n \times n$ matrices $P=P^{T}>0$, $\Omega_{i}=\Omega_{i}^{T}>0, i=1,2, R_{i}=R_{i}^{T}>0, i=1,2, \ldots, 6$, and $S_{i}=S_{i}^{T}>0, i=1,2$, and $\lambda n \times \lambda n$ matrices $Q=Q^{T}>0$ and $(\lambda+5) n \times n$ matrices $N_{i}, i=1,2,3,4$, such that the following set of inequalities hold:

$$
\left[\begin{array}{cccccccc}
\Xi_{11} & \Xi_{12} & \Xi_{13} & \Xi_{14} & \Xi_{15} & \Xi_{16} & \Xi_{17} & \Xi_{18}(l) \\
* & \Xi_{22} & 0_{4 n \times 3 n} & 0_{4 n \times m n} & 0_{4 n \times m n} & 0_{4 n \times m n} & 0_{4 n \times 6 n} & 0_{4 n \times n} \\
* & * & \Xi_{33} & 0_{3 n \times m n} & 0_{3 n \times m n} & 0_{3 n \times m n} & 0_{3 n \times 6 n} & 0_{3 n \times n} \\
* & * & * & \Xi_{44} & 0_{m n \times m n} & 0_{m n \times m n} & 0_{m n \times 6 n} & 0_{m n \times n} \\
* & * & * & * & \Xi_{55} & 0_{m n \times m n} & 0_{m n \times 6 n} & 0_{m n \times n} \\
* & * & * & * & * & \Xi_{66} & 0_{m n \times 6 n} & 0_{m n \times n} \\
* & * & * & * & * & * & \Xi_{77} & 0_{6 n \times n} \\
* & * & * & * & * & * & * & \Xi_{88}
\end{array}\right]
$$

$<0, \quad l=1,2$,

where

$$
\begin{aligned}
& \Xi_{11}=\operatorname{Sym}\left\{E_{1} P \bar{f}_{c}\right\}+W_{\zeta_{1}}^{T} Q W_{\zeta_{1}}-W_{\zeta_{2}}^{T} Q W_{\zeta_{2}} \\
& +E_{1} S_{1} E_{1}^{T}-E_{\lambda+2} S_{1} E_{\lambda+2}^{T}+E_{\lambda+2} S_{2} E_{\lambda+2}^{T} \\
& -E_{\lambda+4} S_{2} E_{\lambda+4}^{T}+\delta\left(E_{\lambda+3}+E_{\lambda+5}\right) \Omega_{1}\left(E_{\lambda+3}^{T}+E_{\lambda+5}^{T}\right) \\
& -E_{\lambda+5} \Omega_{2} E_{\lambda+5}^{T}+\operatorname{Sym}\left\{N_{1}\left(E_{1}^{T}-E_{2}^{T}\right)\right. \\
& +N_{2}\left(E_{1}^{T}-E_{\lambda+2}^{T}\right)+N_{3}\left(E_{\lambda+2}^{T}-E_{\lambda+3}^{T}\right) \\
& \left.+N_{4}\left(E_{\lambda+3}^{T}-E_{\lambda+4}^{T}\right)\right\} \text {, } \\
& \Xi_{12}=\left[g_{c}^{T}, \sqrt{\frac{\tau_{d}}{\lambda}} g_{c}^{T}, \sqrt{\tau_{1}} g_{c}^{T}, \sqrt{\tau_{12}} g_{c}^{T}\right], \\
& \Xi_{22}=\operatorname{diag}\left\{-P^{-1},-R_{2}^{-1},-R_{4}^{-1},-R_{6}^{-1}\right\} \text {, } \\
& \Xi_{13}=\left[\sqrt{\frac{\tau_{d}}{\lambda}} f_{c}^{T}, \sqrt{\tau_{1}} f_{c}^{T}, \sqrt{\tau_{12}} f_{c}^{T}\right], \\
& \Xi_{33}=\operatorname{diag}\left\{-R_{1}^{-1},-R_{3}^{-1},-R_{5}^{-1}\right\} \text {, } \\
& \Xi_{14}=\sqrt{\frac{\tau_{d}}{\lambda}}\left[\beta_{1} \widehat{f}_{1}^{T}, \beta_{2} \widehat{f}_{2}^{T}, \ldots, \beta_{m} \widehat{f}_{m}^{T}\right], \\
& \Xi_{44}=\operatorname{diag}\left\{-R_{1}^{-1},-R_{1}^{-1}, \ldots,-R_{1}^{-1}\right\} \text {, } \\
& \Xi_{15}=\sqrt{\tau_{1}}\left[\beta_{1} \widehat{f}_{1}^{T}, \beta_{2} \widehat{f}_{2}^{T}, \ldots, \beta_{m} \widehat{f}_{m}^{T}\right], \\
& \Xi_{55}=\operatorname{diag}\left\{-R_{3}^{-1},-R_{3}^{-1}, \ldots,-R_{3}^{-1}\right\} \text {, } \\
& \Xi_{16}=\sqrt{\tau_{12}}\left[\beta_{1} \hat{f}_{1}^{T}, \beta_{2} \widehat{f}_{2}^{T}, \ldots, \beta_{m} \hat{f}_{m}^{T}\right], \\
& \Xi_{66}=\operatorname{diag}\left\{-R_{5}^{-1},-R_{5}^{-1}, \ldots,-R_{5}^{-1}\right\} \text {, }
\end{aligned}
$$

$$
\begin{aligned}
& \Xi_{17}=\left[\sqrt{\frac{\tau_{d}}{\lambda}} N_{1}, N_{1}, \sqrt{\tau_{1}} N_{2}, N_{2}, N_{3}, N_{4}\right], \\
& \Xi_{77}=\operatorname{diag}\left\{-R_{1},-R_{2},-R_{3},-R_{4},-R_{6},-R_{6}\right\}, \\
& \Xi_{18}(1)=\sqrt{\tau_{12}} N_{4}, \\
& \Xi_{18}(2)=\sqrt{\tau_{12}} N_{3}, \\
& \Xi_{88}=-R_{5}, \\
& W_{\zeta_{1}}=\left[I_{\lambda n}, 0_{\lambda n \times 5 n}\right], \\
& W_{\zeta_{2}}=\left[0_{\lambda, n \times n}, I_{\lambda n}, 0_{\lambda n \times 4 n}\right], \\
& \bar{f}_{c}=A E_{1}^{T}+A_{d} E_{\lambda+1}^{T}+B \bar{\Lambda} K E_{\lambda+3}^{T}+B \bar{\Lambda} K E_{\lambda+5}^{T}, \\
& \hat{f}_{i}=B e_{i} K E_{\lambda+3}^{T}+B e_{i} K E_{\lambda+5}^{T}, \\
& g_{c}=H E_{1}^{T}+H_{d} E_{\lambda+1}^{T} .
\end{aligned}
$$

Proof. For the sake of notational convenience, let

$$
\begin{aligned}
& f(t)=\bar{f}(t)+\hat{f}(t), \\
& g(t)=H x(t)+H_{d} x\left(t-\tau_{d}\right),
\end{aligned}
$$

where

$$
\begin{aligned}
\bar{f}(t)= & A x(t)+A_{d} x\left(t-\tau_{d}\right)+B \bar{\Lambda} K x(t-\tau(t)) \\
& +B \bar{\Lambda} K e(t), \\
\widehat{f}(t)= & B(\Lambda-\bar{\Lambda}) K x(t-\tau(t))+B(\Lambda-\bar{\Lambda}) K e(t) .
\end{aligned}
$$

Then, we can recast (13) into following form:

$$
d(t)=f(t) d t+g(t) d \omega(t) .
$$

We divide the time-delay interval $\left[t-\tau_{d}, t\right]$ into $\lambda$ uniform subintervals and define the following augmented variable:

$$
\begin{aligned}
& \varsigma^{T}(t) \\
& \quad=\left[x^{T}(t), x^{T}\left(t-\frac{\tau_{d}}{\lambda}\right), \ldots, x^{T}\left(t-(\lambda-1) \frac{\tau_{d}}{\lambda}\right)\right] .
\end{aligned}
$$

Choose the following simple functional candidate for system (21):

$$
V(x(t), t)=\sum_{i=1}^{4} V_{i}(x(t), t),
$$


where $x(t)$ denotes the function $x(s)$ defined on the interval $\left[t-\max \left\{\tau_{d}, \tau_{2}\right\}, t\right]$, and

$$
\begin{aligned}
& V_{1}(x(t), t)=x^{T}(t) P x(t), \\
& V_{2}(x(t), t)=\int_{t-\tau_{d} / \lambda}^{t}\left[\varsigma^{T}(s) Q \varsigma(s)+\left(\frac{\tau_{d}}{\lambda}-t+s\right)\right. \\
& \left.\cdot\left(f^{T}(s) R_{1} f(s)+g^{T}(s) R_{2} g(s)\right)\right] d s, \\
& V_{3}(x(t), t)=\int_{t-\tau_{1}}^{t}\left[x^{T}(s) S_{1} x(s)+\left(\tau_{1}-t+s\right)\right. \\
& \left.\cdot\left(f^{T}(s) R_{3} f(s)+g^{T}(s) R_{4} g(s)\right)\right] d s, \\
& V_{4}(x(t), t)=\int_{t-\tau_{1}}^{t} \tau_{12}\left(f^{T}(s) R_{5} f(s)+g^{T}(s)\right. \\
& \left.\cdot R_{6} g(s)\right) d s+\int_{t-\tau_{2}}^{t-\tau_{1}}\left[x^{T}(s) S_{2} x(s)\right. \\
& +\left(\tau_{2}-t+s\right) \\
& \left.\quad\left(f^{T}(s) R_{5} f(s)+g^{T}(s) R_{6} g(s)\right)\right] d s .
\end{aligned}
$$

For the sake of notational simplification, let

$$
\begin{aligned}
& \zeta_{t}^{T}= {\left[\varsigma^{T}(t), x^{T}\left(t-\tau_{d}\right), x^{T}\left(t-\tau_{1}\right), x^{T}(t-\tau(t)),\right.} \\
&\left.x^{T}\left(t-\tau_{2}\right), e^{T}(t)\right], \\
& E_{s}= {[\underbrace{0_{n \times n}, \ldots, 0_{n \times n},}_{s-1} I_{n \times n}, \underbrace{0_{n \times n}, \ldots, 0_{n \times n}}_{\lambda+5-s}]^{T}, } \\
& s=1,2, \ldots, \lambda+5, \\
& \bar{f}_{c}=A E_{1}^{T}+A_{d} E_{\lambda+1}^{T}+B \bar{\Lambda} K E_{\lambda+3}^{T}+B \bar{\Lambda} K E_{\lambda+5}^{T}, \\
& \hat{f}_{i}=B e_{i} K E_{\lambda+3}^{T}+B e_{i} K E_{\lambda+5}^{T}, \\
& g_{c}=H E_{1}^{T}+H_{d} E_{\lambda+1}^{T} .
\end{aligned}
$$

Taking the time derivatives of $V_{i}(x(t), t), i=1,2,3,4$, along the trajectory of system (21) and taking expectation on it yield

$$
\begin{aligned}
E\{ & \left.d V_{1}(x(t), t)\right\}=E\left\{2 x^{T}(t) P f(t) d t\right. \\
& \left.+g^{T}(t) P g(t) d t+2 x^{T}(t) P g(t) d \omega(t)\right\} \\
& =\zeta_{t}^{T}\left\{2 E_{1} P \bar{f}_{c} d t+g_{c}^{T} P g_{c} d t\right\} \zeta_{t}, \\
E & \left\{\dot{V}_{2}(x(t), t)\right\}=\zeta_{t}^{T}\left\{W_{\zeta_{1}}^{T} Q W_{\zeta_{1}}-W_{\zeta_{2}}^{T} Q W_{\zeta_{2}}\right. \\
& \left.+\frac{\tau_{d}}{\lambda} g_{c}^{T} R_{2} g_{c}\right\} \zeta_{t}+E\left\{\frac{\tau_{d}}{\lambda} f^{T}(t) R_{1} f(t)\right.
\end{aligned}
$$

$$
\begin{aligned}
& -\int_{t-\tau_{d} / \lambda}^{t} f^{T}(s) R_{1} f(s) d s \\
& \left.-\int_{t-\tau_{d} / \lambda}^{t} g^{T}(s) R_{2} g(s) d s\right\} \\
E & \left\{\dot{V}_{3}(x(t), t)\right\}=\zeta_{t}^{T}\left\{E_{1} S_{1} E_{1}^{T}-E_{\lambda+2} S_{1} E_{\lambda+2}^{T}\right. \\
& \left.+\tau_{1} g_{c}^{T} R_{4} g_{c}\right\} \zeta_{t}+E\left\{\tau_{1} f^{T}(t) R_{3} f(t)\right. \\
& \left.-\int_{t-\tau_{1}}^{t} f^{T}(s) R_{3} f(s) d s-\int_{t-\tau_{1}}^{t} g^{T}(s) R_{4} g(s) d s\right\}, \\
E\{ & \left.\dot{V}_{4}(x(t), t)\right\}=\zeta_{t}^{T}\left\{E_{\lambda+2} S_{2} E_{\lambda+2}^{T}-E_{\lambda+4} S_{2} E_{\lambda+4}^{T}\right. \\
& \left.+\tau_{12} g_{c}^{T}(t) R_{6} g_{c}\right\} \zeta_{t}+E\left\{\tau_{12} f^{T}(t) R_{5} f(t)\right. \\
& \left.-\int_{t-\tau_{2}}^{t-\tau_{1}} f^{T}(s) R_{5} f(s) d s-\int_{t-\tau_{2}}^{t-\tau_{1}} g^{T}(s) R_{6} g(s) d s\right\},
\end{aligned}
$$

where $\tau_{12} \triangleq \tau_{2}-\tau_{1}$.

The calculation shows that

$$
\begin{aligned}
E & \left\{\frac{\tau_{d}}{\lambda} f^{T}(t) R_{1} f(t)+\tau_{1} f^{T}(t) R_{3} f(t)+\tau_{12} f^{T}(t)\right. \\
& \left.\cdot R_{5} f(t)\right\} \\
& =E\left\{\zeta _ { t } ^ { T } \left\{\bar{f}_{c}^{T}\left(\frac{\tau_{d}}{\lambda} R_{1}+\tau_{1} R_{3}+\tau_{12} R_{5}\right) \bar{f}_{c}\right.\right. \\
& \left.\left.+\sum_{i=1}^{m} \beta_{i}^{2} \widehat{f}_{i}^{T}\left(\frac{\tau_{d}}{\lambda} R_{1}+\tau_{1} R_{3}+\tau_{12} R_{5}\right) \hat{f}_{i}\right\} \zeta_{t}\right\} .
\end{aligned}
$$

According to the Newton-Leibniz formula, the inner relationship among the terms in the Leibniz-Newton formula is revealed by introducing free weighting matrices $N_{i}, i=$ $1,2,3,4$. Then, the following terms are true:

$$
\begin{aligned}
& 2 \zeta_{t}^{T} N_{1}\left(x(t)-x\left(t-\frac{\tau_{d}}{\lambda}\right)-\int_{t-\tau_{d} / \lambda}^{t} f(s) d s\right. \\
& \left.-\int_{t-\tau_{d} / \lambda}^{t} g(s) d \Phi(s)\right) \equiv 0, \\
& 2 \zeta_{t}^{T} N_{2}\left(x(t)-x\left(t-\tau_{1}\right)-\int_{t-\tau_{1}}^{t} f(s) d s\right. \\
& \left.\quad-\int_{t-\tau_{1}}^{t} g(s) d \omega(s)\right) \equiv 0, \\
& 2 \zeta_{t}^{T} N_{3}\left(x\left(t-\tau_{1}\right)-x(t-\tau(t))-\int_{t-\tau(t)}^{t-\tau_{1}} f(s) d s\right. \\
& \left.\quad-\int_{t-\tau(t)}^{t-\tau_{1}} g(s) d \omega(s)\right) \equiv 0,
\end{aligned}
$$


6

Scientific Programming

$$
\begin{aligned}
2 \zeta_{t}^{T} & N_{4}\left(x(t-\tau(t))-x\left(t-\tau_{2}\right)-\int_{t-\tau_{2}}^{t-\tau(t)} f(s) d s\right. \\
- & \left.\int_{t-\tau_{2}}^{t-\tau(t)} g(s) d \omega(s)\right) \equiv 0 .
\end{aligned}
$$

Moreover, from the well-known fact, $-2 a^{T} b \leq a^{T} R_{i}^{-1} a+$ $b^{T} R_{i} b, i=1,2, \ldots, 6$, for two appropriate dimensional rectors $a$ and $b$, it can be easily checked that

$$
\begin{aligned}
& 2 \zeta_{t}^{T} N_{1}\left(-\int_{t-\tau_{d} / \lambda}^{t} f(s) d s-\int_{t-\tau_{d} / \lambda}^{t} g(s) d \omega(s)\right) \\
& \leq \zeta_{t}^{T}\left(\frac{\tau_{d}}{\lambda} N_{1} R_{1}^{-1} N_{1}^{T}+N_{1} R_{2}^{-1} N_{1}^{T}\right) \zeta_{t} \\
& +\int_{t-\tau_{d} / \lambda}^{t} f^{T}(s) R_{1} f(s) d s \\
& +\left(\int_{t-\tau_{d} / \lambda}^{t} g(s) d \omega(t)\right)^{T} R_{2}\left(\int_{t-\tau_{d} / \lambda}^{t} g(s) d \omega(t)\right), \\
& 2 \zeta_{t}^{T} N_{2}\left(-\int_{t-\tau_{1}}^{t} f(s) d s-\int_{t-\tau_{1}}^{t} g(s) d \omega(s)\right) \\
& \leq \zeta_{t}^{T}\left(\tau_{1} N_{2} R_{3}^{-1} N_{2}^{T}+N_{2} R_{4}^{-1} N_{2}^{T}\right) \zeta_{t} \\
& +\int_{t-\tau_{1}}^{t} f^{T}(s) R_{3} f(s) d s+\left(\int_{t-\tau_{1}}^{t} g(s) d \omega(t)\right)^{T} \\
& \cdot R_{4}\left(\int_{t-\tau_{1}}^{t} g(s) d \omega(t)\right) \\
& 2 \zeta_{t}^{T} N_{3}\left(-\int_{t-\tau(t)}^{t-\tau_{1}} f(s) d s-\int_{t-\tau(t)}^{t-\tau_{1}} g(s) d \omega(s)\right) \\
& \leq \zeta_{t}^{T}\left(\left(\tau(t)-\tau_{1}\right) N_{3} R_{5}^{-1} N_{3}^{T}+N_{3} R_{6}^{-1} N_{3}^{T}\right) \zeta_{t} \\
& +\int_{t-\tau(t)}^{t-\tau_{1}} f^{T}(s) R_{5} f(s) d s+\left(\int_{t-\tau(t)}^{t-\tau_{1}} g(s) d \omega(t)\right)^{T} \\
& \cdot R_{6}\left(\int_{t-\tau(t)}^{t-\tau_{1}} g(s) d \omega(t)\right) \\
& 2 \zeta_{t}^{T} N_{4}\left(-\int_{t-\tau_{2}}^{t-\tau(t)} f(s) d s-\int_{t-\tau_{2}}^{t-\tau(t)} g(s) d \omega(s)\right) \\
& \leq \zeta_{t}^{T}\left(\left(\tau_{2}-\tau(t)\right) N_{4} R_{5}^{-1} N_{4}^{T}+N_{4} R_{6}^{-1} N_{4}^{T}\right) \zeta_{t} \\
& +\int_{t-\tau(t)}^{t-\tau_{1}} f^{T}(s) R_{5} f(s) d s+\left(\int_{t-\tau_{2}}^{t-\tau(t)} g(s) d \omega(t)\right)^{T} \\
& \cdot R_{6}\left(\int_{t-\tau_{2}}^{t-\tau(t)} g(s) d \omega(t)\right),
\end{aligned}
$$

as

$$
\begin{aligned}
& E\left\{\left(\int_{t-\tau_{d} / \lambda}^{t} g(s) d \omega(t)\right)^{T} R_{2}\left(\int_{t-\tau_{d} / \lambda}^{t} g(s) d \omega(t)\right)\right\} \\
& \quad=E\left\{\int_{t-\tau_{d} / \lambda}^{t} g^{T}(s) R_{2} g(s) d s\right\}, \\
& E\left\{\left(\int_{t-\tau_{1}}^{t} g(s) d \omega(t)\right)^{T} R_{4}\left(\int_{t-\tau_{1}}^{t} g(s) d \omega(t)\right)\right\} \\
& \quad=E\left\{\int_{t-\tau_{1}}^{t} g^{T}(s) R_{4} g(s) d s\right\}, \\
& E\left\{\left(\int_{t-\tau(t)}^{t-\tau_{1}} g(s) d \omega(t)\right)^{T} R_{6}\left(\int_{t-\tau(t)}^{t-\tau_{1}} g(s) d \omega(t)\right)\right\} \\
& \quad=E\left\{\int_{t-\tau(t)}^{t-\tau_{1}} g^{T}(s) R_{6} g(s) d s\right\}, \\
& E\left\{\left(\int_{t-\tau_{2}}^{t-\tau(t)} g(s) d \omega(t)\right)^{T} R_{6}\left(\int_{t-\tau_{2}}^{t-\tau(t)} g(s) d \omega(t)\right)\right\} \\
& \quad=E\left\{\int_{t-\tau_{2}}^{t-\tau(t)} g^{T}(s) R_{6} g(s) d s\right\} .
\end{aligned}
$$

Combining (26)-(30) yields

$$
\begin{aligned}
& \dot{V}(x(t), t) \leq \zeta_{t}^{T}\left\{2 E_{1} P \bar{f}_{c}+g_{c}^{T} P g_{c}+W_{\varsigma_{1}}^{T} Q W_{\varsigma_{1}}\right. \\
& -W_{\varsigma_{2}}^{T} Q W_{\varsigma_{2}}+\frac{\tau_{d}}{\lambda} g_{c}^{T} R_{2} g_{c}+\frac{\tau_{d}}{\lambda} \bar{f}_{c}^{T} R_{1} \bar{f}_{c} \\
& +\frac{\tau_{d}}{\lambda} \sum_{i=1}^{m} \beta_{i}^{2} \widehat{f}_{i}^{T} R_{1} \widehat{f}_{i}+E_{1} S_{1} E_{1}^{T}-E_{\lambda+2} S_{1} E_{\lambda+2}^{T} \\
& +\tau_{1} g_{c}^{T} R_{4} g_{c}+\tau_{1} \bar{f}_{c}^{T} R_{3} \bar{f}_{c}+\tau_{1} \sum_{i=1}^{m} \beta_{i}^{2} \widehat{f}_{i}^{T} R_{3} \widehat{f}_{i} \\
& +E_{\lambda+2} S_{2} E_{\lambda+2}^{T}-E_{\lambda+4} S_{2} E_{\lambda+4}^{T}+\tau_{12} g_{c}^{T} R_{6} g_{c} \\
& +\tau_{12} \bar{f}_{c}^{T} R_{5} \bar{f}_{c}+\tau_{12} \sum_{i=1}^{m} \beta_{i}^{2} \widehat{f}_{i}^{T} R_{5} \widehat{f}_{i} \\
& +\delta\left(E_{\lambda+3}+E_{\lambda+5}\right) \Omega_{1}\left(E_{\lambda+3}+E_{\lambda+5}\right)^{T} \\
& -E_{\lambda+5} \Omega_{2} E_{\lambda+5}^{T}+2 N_{1}\left(E_{1}^{T}-E_{2}^{T}\right) \\
& +2 N_{2}\left(E_{1}^{T}-E_{\lambda+2}^{T}\right)+2 N_{3}\left(E_{\lambda+2}^{T}-E_{\lambda+3}^{T}\right) \\
& +2 N_{4}\left(E_{\lambda+3}^{T}-E_{\lambda+4}^{T}\right)+\frac{\tau_{d}}{\lambda} N_{1} R_{1}^{-1} N_{1}^{T}+N_{1} R_{2}^{-1} N_{1}^{T} \\
& +\tau_{1} N_{2} R_{3}^{-1} N_{2}^{T}+N_{2} R_{4}^{-1} N_{2}^{T} \\
& +\left(\tau(t)-\tau_{1}\right) N_{3} R_{5}^{-1} N_{3}^{T}+N_{3} R_{6}^{-1} N_{3}^{T} \\
& \left.+\left(\tau_{2}-\tau(t)\right) N_{4} R_{5}^{-1} N_{4}^{T}+N_{4} R_{6}^{-1} N_{4}^{T}\right\} \zeta_{t}+e^{T}(t)
\end{aligned}
$$




$$
\begin{aligned}
& \cdot \Omega_{2} e(t)-\delta(e(t)+x(t-\tau(t)))^{T} \Omega_{1}(e(t) \\
& +x(t-\tau(t)))=\zeta_{t}^{T} \Xi(\tau(t)) \zeta_{t}+e^{T}(t) \Omega_{2} e(t) \\
& -\delta(e(t)+x(t-\tau(t)))^{T} \Omega_{1}(e(t)+x(t-\tau(t))),
\end{aligned}
$$

where

$$
\Xi(\tau(t))=\Xi_{11}+\Xi_{12}(\tau(t)), \quad \tau(t) \in\left[\tau_{1}, \tau_{2}\right] .
$$

$\Xi_{11}$ is defined in Theorem 8 , and

$$
\begin{aligned}
\Xi_{12}(\tau(t))= & g_{c}^{T} P g_{c}+\frac{\tau_{d}}{\lambda} g_{c}^{T} R_{2} g_{c}+\frac{\tau_{d}}{\lambda} \bar{f}_{c}^{T} R_{1} \bar{f}_{c} \\
& +\frac{\tau_{d}}{\lambda} \sum_{i=1}^{m} \beta_{i}^{2} \widehat{f}_{i}^{T} R_{1} \widehat{f}_{i}+\tau_{1} g_{c}^{T} R_{4} g_{c} \\
& +\tau_{1} \bar{f}_{c}^{T} R_{3} \bar{f}_{c}+\tau_{1} \sum_{i=1}^{m} \beta_{i}^{2} \widehat{f}_{i}^{T} R_{3} \widehat{f}_{i} \\
& +\tau_{12} g_{c}^{T} R_{6} g_{c}+\tau_{12} \bar{f}_{c}^{T} R_{5} \bar{f}_{c} \\
& +\tau_{12} \sum_{i=1}^{m} \beta_{i}^{2} \widehat{f}_{i}^{T} R_{5} \widehat{f}_{i}+\frac{\tau_{d}}{\lambda} N_{1} R_{1}^{-1} N_{1}^{T} \\
& +N_{1} R_{2}^{-1} N_{1}^{T}+\tau_{1} N_{2} R_{3}^{-1} N_{2}^{T}+N_{2} R_{4}^{-1} N_{2}^{T} \\
& +\left(\tau(t)-\tau_{1}\right) N_{3} R_{5}^{-1} N_{3}^{T}+N_{3} R_{6}^{-1} N_{3}^{T} \\
& +\left(\tau_{2}-\tau(t)\right) N_{4} R_{5}^{-1} N_{4}^{T}+N_{4} R_{6}^{-1} N_{4}^{T} .
\end{aligned}
$$

From (31)-(33), it is not difficult to see that $\zeta_{t}^{T} \Xi(\tau(t)) \zeta_{t}$ is a first-order function on $\tau(t)$, and the first-order coefficient is $N_{3} R_{5}^{-1} N_{3}^{T}-N_{4} R_{5}^{-1} N_{4}^{T}$. According to Lemma 6, the condition $\Xi(\tau(t))<0$ is equivalent to

$$
\begin{aligned}
& \Xi_{11}+\Xi_{12}\left(\tau_{1}\right)<0, \\
& \Xi_{11}+\Xi_{12}\left(\tau_{2}\right)<0 .
\end{aligned}
$$

Consequently, further resorting to the Schur complement lemma [30], inequalities (17) can be obtained.

In the following, we will show that stochastic system (21) satisfies event-triggered algorithm (4). Integrate on both sides of inequality (31) from 0 to $t$, and according to $V(0)=0$, $V(t) \geq 0$, we can learn that if inequalities (17) are feasible, then

$$
\begin{aligned}
& e^{T}(t) \Omega_{2} e(t) \\
& \quad \geq \delta(e(t)+x(t-\tau(t)))^{T} \Omega_{1}(e(t)+x(t-\tau(t)))
\end{aligned}
$$

holds for arbitrary $t \in\left[t_{k} h+\tau_{t_{k}}, t_{k+1} h+\tau_{t_{k+1}}\right]$.

This completes the proof.

The sufficient condition that stochastic system (13) with time-delay satisfying condition (7) is stochastically asymptotically stable is given in Theorem 8 with coupled matrix variables, but the controller gain matrix cannot be obtained directly. So, based on Theorem 8 , we next prove a new theorem for the event-triggered controller design in terms of linear matrix inequalities through congruent transformation.

Theorem 9. For given scalars $\tau_{d}, \tau_{1}, \tau_{2}$, and $\delta$ and a positive integer $\lambda$, the closed-loop stochastic system with time delays (13) and time-varying delay satisfying condition (7) is stochastically asymptotically stable if there exist $n \times n$ matrices $X=X^{T}>0$, $\widetilde{\Omega}_{i}=\widetilde{\Omega}_{i}^{T}>0, i=1,2, \widetilde{R}_{i}=\widetilde{R}_{i}^{T}>0, i=1,2, \ldots, 6$, and $\widetilde{S}_{i}=\widetilde{S}_{i}^{T}>0, i=1,2$, and $\lambda n \times \lambda n$ matrices $\widetilde{Q}=\widetilde{Q}^{T}>0$ and $(\lambda+5) n \times n$ matrices $\widetilde{N}_{i}, i=1,2,3,4$, such that the following set of inequalities hold:

$$
\left[\begin{array}{cccccccc}
\widetilde{\Xi}_{11} & \widetilde{\Xi}_{12} & \widetilde{\Xi}_{13} & \widetilde{\Xi}_{14} & \widetilde{\Xi}_{15} & \widetilde{\Xi}_{16} & \widetilde{\Xi}_{17} & \widetilde{\Xi}_{18}(l) \\
* & \widetilde{\Xi}_{22} & 0_{4 n \times 3 n} & 0_{4 n \times m n} & 0_{4 n \times m n} & 0_{4 n \times m n} & 0_{4 n \times 6 n} & 0_{4 n \times n} \\
* & * & \widetilde{\Xi}_{33} & 0_{3 n \times m n} & 0_{3 n \times m n} & 0_{3 n \times m n} & 0_{3 n \times 6 n} & 0_{3 n \times n} \\
* & * & * & \widetilde{\Xi}_{44} & 0_{m n \times m n} & 0_{m n \times m n} & 0_{m n \times 6 n} & 0_{m n \times n} \\
* & * & * & * & \widetilde{\Xi}_{55} & 0_{m n \times m n} & 0_{m n \times 6 n} & 0_{m n \times n} \\
* & * & * & * & * & \widetilde{\Xi}_{66} & 0_{m n \times 6 n} & 0_{m n \times n} \\
* & * & * & * & * & * & \widetilde{\Xi}_{77} & 0_{6 n \times n} \\
* & * & * & * & * & * & * & \widetilde{\Xi}_{88}
\end{array}\right]
$$

$<0, \quad l=1,2$,

where

$$
\begin{aligned}
& \widetilde{\Xi}_{11}=\operatorname{Sym}\left\{E_{1} \widetilde{f}_{c}\right\}+W_{\zeta_{1}}^{T} \widetilde{Q} W_{\zeta_{1}}-W_{\zeta_{2}}^{T} \widetilde{Q} W_{\zeta_{2}}+E_{1} \widetilde{S}_{1} E_{1}^{T} \\
& -E_{\lambda+2} \widetilde{S}_{1} E_{\lambda+2}^{T}+E_{\lambda+2} \widetilde{S}_{2} E_{\lambda+2}^{T}-E_{\lambda+4} \widetilde{S}_{2} E_{\lambda+4}^{T} \\
& +\delta\left(E_{\lambda+3}+E_{\lambda+5}\right) \widetilde{\Omega}_{1}\left(E_{\lambda+3}^{T}+E_{\lambda+5}^{T}\right)-E_{\lambda+5} \widetilde{\Omega}_{2} E_{\lambda+5}^{T} \\
& +\operatorname{Sym}\left\{\widetilde{N}_{1}\left(E_{1}^{T}-E_{2}^{T}\right)+\widetilde{N}_{2}\left(E_{1}^{T}-E_{\lambda+2}^{T}\right)\right. \\
& \left.+\widetilde{N}_{3}\left(E_{\lambda+2}^{T}-E_{\lambda+3}^{T}\right)+\widetilde{N}_{4}\left(E_{\lambda+3}^{T}-E_{\lambda+4}^{T}\right)\right\}, \\
& \widetilde{\Xi}_{12}=\left[\tilde{g}_{c}^{T}, \sqrt{\frac{\tau_{d}}{\lambda}} \tilde{g}_{c}^{T}, \sqrt{\tau_{1}} \widetilde{g}_{c}^{T}, \sqrt{\tau_{12}} \widetilde{g}_{c}^{T}\right], \\
& \widetilde{\Xi}_{22}=\operatorname{diag}\left\{-X,-2 X+\widetilde{R}_{2},-2 X+\widetilde{R}_{4},-2 X+\widetilde{R}_{6}\right\}, \\
& \widetilde{\Xi}_{13}=\left[\sqrt{\frac{\tau_{d}}{\lambda}} \widetilde{f}_{c}^{T}, \sqrt{\tau_{1}} \tilde{f}_{c}^{T}, \sqrt{\tau_{12}} \widetilde{f}_{c}^{T}\right], \\
& \widetilde{\Xi}_{33}=\operatorname{diag}\left\{-2 X+\widetilde{R}_{1},-2 X+\widetilde{R}_{3},-2 X+\widetilde{R}_{5}\right\} \text {, } \\
& \widetilde{\Xi}_{14}=\sqrt{\frac{\overline{\tau_{d}}}{\lambda}}\left[\beta_{1} \breve{f}_{1}^{T}, \beta_{2} \breve{f}_{2}^{T}, \ldots, \beta_{m} \breve{f}_{m}^{T}\right], \\
& \widetilde{\Xi}_{44}=\operatorname{diag}\left\{-2 X+\widetilde{R}_{1},-2 X+\widetilde{R}_{1}, \ldots,-2 X+\widetilde{R}_{1}\right\} \text {, } \\
& \widetilde{\Xi}_{15}=\sqrt{\tau_{1}}\left[\beta_{1} \breve{f}_{1}^{T}, \beta_{2} \breve{f}_{2}^{T}, \ldots, \beta_{m} \breve{f}_{m}^{T}\right], \\
& \widetilde{\Xi}_{55}=\operatorname{diag}\left\{-2 X+\widetilde{R}_{3},-2 X+\widetilde{R}_{3}, \ldots,-2 X+\widetilde{R}_{3}\right\} \text {, } \\
& \widetilde{\Xi}_{16}=\sqrt{\tau_{12}}\left[\beta_{1} \breve{f}_{1}^{T}, \beta_{2} \breve{f}_{2}^{T}, \ldots, \beta_{m} \breve{f}_{m}^{T}\right],
\end{aligned}
$$




$$
\begin{aligned}
& \widetilde{\Xi}_{66}=\operatorname{diag}\left\{-2 X+\widetilde{R}_{5},-2 X+\widetilde{R}_{5}, \ldots,-2 X+\widetilde{R}_{5}\right\}, \\
& \widetilde{\Xi}_{17}=\left[\sqrt{\frac{\tau_{d}}{\lambda}} \widetilde{N}_{1}, \widetilde{N} 1, \sqrt{\tau_{1}} \widetilde{N}_{2}, \widetilde{N}_{2}, \widetilde{N}_{3}, \widetilde{N}_{4}\right], \\
& \widetilde{\Xi}_{77}=\operatorname{diag}\left\{-\widetilde{R}_{1},-\widetilde{R}_{2},-\widetilde{R}_{3},-\widetilde{R}_{4},-\widetilde{R}_{6},-\widetilde{R}_{6}\right\}, \\
& \widetilde{\Xi}_{18}(1)=\sqrt{\tau_{12}} \widetilde{N}_{4}, \\
& \widetilde{\Xi}_{18}(2)=\sqrt{\tau_{12}} \widetilde{N}_{3}, \\
& \widetilde{\Xi}_{88}=-\widetilde{R}_{5}, \\
& W_{\zeta_{1}}=\left[I_{\lambda n}, 0_{\lambda n \times 5 n}\right], \\
& W_{\zeta_{2}}=\left[00_{\lambda n \times n}, I_{\lambda n}, 0_{\lambda n \times 4 n}\right], \\
& \widetilde{f}_{c}=A X E_{1}^{T}+A_{d} X E_{\lambda+1}^{T}+B \bar{\Lambda} \widehat{K} E_{\lambda+3}^{T}+B \bar{\Lambda} \widehat{K} E_{\lambda+5}^{T}, \\
& \breve{f}_{i}=B e_{i} \widehat{K} E_{\lambda+3}^{T}+B e_{i} \widehat{K} E_{\lambda+5}^{T}, \\
& \widetilde{g}_{c}=H X E_{1}^{T}+H_{d} X E_{\lambda+1}^{T}
\end{aligned}
$$

and a desired controller gain matrix in (2) can be chosen as

$$
K=\widehat{K} X^{-1}
$$

Proof. Define

$$
J=\operatorname{diag}\left\{J_{1}, J_{2}, J_{3}, J_{4}, J_{4}, J_{4}, J_{5}, P^{-1}\right\},
$$

where

$$
\begin{aligned}
& J_{1}=\operatorname{diag}\left\{P^{-1}, P^{-1}, \ldots, P^{-1}\right\} \in \mathbb{R}^{(\lambda+5) n}, \\
& J_{2}=\operatorname{diag}\{I, I, I, I\}, \\
& J_{3}=\operatorname{diag}\{I, I, I\}, \\
& J_{4}=\operatorname{diag}\{I, I, \ldots, I\} \in \mathbb{R}^{m n}, \\
& J_{5}=\operatorname{diag}\left\{P^{-1}, P^{-1}, P^{-1}, P^{-1}, P^{-1}, P^{-1}\right\} .
\end{aligned}
$$

Pre- and postmultiplying (17) with $J^{T}$ and $J$, noticing that

$$
\begin{array}{r}
R_{i}^{-1}-2 X+X R_{i} X=\left(R_{i}^{-1}-X\right) R_{i}\left(R_{i}^{-1}-X\right) \geq 0 \\
i=1,2, \ldots, 6
\end{array}
$$

is equivalent to

$$
-R_{i}^{-1} \leq-2 X+X R_{i} X, \quad i=1,2, \ldots, 6
$$

we introduce the following new matrix variables:

$$
\begin{aligned}
& X=P^{-1} ; \\
& \widetilde{R}_{i}=X R_{i}^{T} X, \quad i=1,2, \ldots, 6 ; \\
& \widetilde{S}_{i}=X S_{i}^{T} X, \quad i=1,2, \\
& \widetilde{Q}=J_{6}^{T} Q J_{6}, \quad J_{6}=\operatorname{diag}\{X, X, \ldots, X\} \in \mathbb{R}^{\lambda n} ; \\
& \widetilde{\Omega}_{i}=X \Omega_{i}^{T} X, \quad i=1,2, \\
& \widehat{K}^{\prime}=K X ; \\
& \widetilde{N}_{i}=J_{1} N_{i} X, \quad i=1,2,3,4, \\
& \widetilde{f}_{c}=A X E_{1}^{T}+A_{d} X E_{\lambda+1}^{T}+B \bar{\Lambda} \widehat{K} E_{\lambda+3}^{T}+B \bar{\Lambda} \widehat{K} E_{\lambda+5}^{T}, \\
& \breve{f}_{i}=B e_{i} \widehat{K} E_{\lambda+3}^{T}+B e_{i} \widehat{K} E_{\lambda+5}^{T}, \\
& \widetilde{g}_{c}=H X E_{1}^{T}+H H_{d} X E_{\lambda+1}^{T},
\end{aligned}
$$

and then, we can learn that (36) is equivalent to (17).

This completes the proof.

Remark 10. It is worth mentioning that Theorem 9 is also applicable to the event-triggered faults tolerant control for system (1) without state-delay and stochastic perturbations. The proof follows a similar line to Theorems 8 and 9. Now we formally present this result as the following corollary.

Corollary 11. For given scalars $\tau_{1}$ and $\tau_{2}$ and $0 \leq \delta<1$, the closed-loop system with time delays (13) and time-varying delay satisfying condition (7) is asymptotically stable if there exist $n \times$ $n$ matrices $X=X^{T}>0, \widetilde{\Omega}_{i}=\widetilde{\Omega}_{i}^{T}>0, i=1,2, \widetilde{R}_{i}=\widetilde{R}_{i}^{T}>$ $0, i=3,5$, and $\widetilde{S}_{i}=\widetilde{S}_{i}^{T}>0, i=1,2$, and $(\lambda+5) n \times n$ matrices $\widetilde{N}_{i}, i=1,2,3$, such that the following set of inequalities hold:

$$
\left[\begin{array}{cccccc}
\widetilde{\Xi}_{11} & \widetilde{\Xi}_{12} & \widetilde{\Xi}_{13} & \widetilde{\Xi}_{14} & \widetilde{\Xi}_{15} & \widetilde{\Xi}_{16}(l) \\
* & \widetilde{\Xi}_{22} & 0_{2 n \times 2 n} & 0_{2 n \times 2 n} & 0_{2 n \times n} & 0_{2 n \times n} \\
* & * & \widetilde{\Xi}_{33} & 0_{2 n \times 2 n} & 0_{2 n \times n} & 0_{2 n \times n} \\
* & * & * & \widetilde{\Xi}_{44} & 0_{2 n \times n} & 0_{2 n \times n} \\
* & * & * & * & \widetilde{\Xi}_{55} & 0_{n \times n} \\
* & * & * & * & * & \widetilde{\Xi}_{66}
\end{array}\right]<0,
$$

$l=1,2$,

where

$$
\begin{aligned}
\widetilde{\Xi}_{11} & =\operatorname{Sym}\left\{E_{1} \widetilde{A}_{c}\right\}+E_{1} \widetilde{S}_{1} E_{1}^{T}-E_{2} \widetilde{S}_{1} E_{2}^{T}+E_{2} \widetilde{S}_{2} E_{2}^{T} \\
& -E_{4} \widetilde{S}_{2} E_{4}^{T}+\delta\left(E_{3}+E_{5}\right) \widetilde{\Omega}_{1}\left(E_{3}^{T}+E_{5}^{T}\right)-E_{5} \widetilde{\Omega}_{2} E_{5}^{T} \\
& +\operatorname{Sym}\left\{\widetilde{N}_{1}\left(E_{1}^{T}-E_{2}^{T}\right)+\widetilde{N}_{2}\left(E_{2}^{T}-E_{3}^{T}\right)\right. \\
& \left.+\widetilde{N}_{3}\left(E_{3}^{T}-E_{4}^{T}\right)\right\},
\end{aligned}
$$




$$
\begin{aligned}
& \widetilde{\Xi}_{12}=\left[\sqrt{\tau_{1}} \widetilde{A}_{c}^{T}, \sqrt{\tau_{12}} \widetilde{A}_{c}^{T}\right], \\
& \widetilde{\Xi}_{22}=\operatorname{diag}\left\{-2 X+\widetilde{R}_{3},-2 X+\widetilde{R}_{5}\right\}, \\
& \widetilde{\Xi}_{13}=\sqrt{\tau_{1}}\left[\beta_{1} \breve{A}_{c 1}^{T}, \beta_{2} \breve{A}_{c 2}^{T}, \ldots, \beta_{m} \breve{A}_{c m}^{T}\right], \\
& \widetilde{\Xi}_{33}=\operatorname{diag}\left\{-2 X+\widetilde{R}_{3},-2 X+\widetilde{R}_{3}, \ldots,-2 X+\widetilde{R}_{3}\right\}, \\
& \widetilde{\Xi}_{14}=\sqrt{\tau_{12}}\left[\beta_{1} \breve{A}_{c 1}^{T}, \beta_{2} \breve{A}_{c 2}^{T}, \ldots, \beta_{m} \breve{A}_{c m}^{T}\right], \\
& \widetilde{\Xi}_{44}=\operatorname{diag}\left\{-2 X+\widetilde{R}_{5},-2 X+\widetilde{R}_{5}, \ldots,-2 X+\widetilde{R}_{5}\right\}, \\
& \widetilde{\Xi}_{15}=\sqrt{\tau_{1}} \widetilde{N}_{1}, \\
& \widetilde{\Xi}_{55}=-\widetilde{R}_{3}, \\
& \widetilde{\Xi}_{16}(1)=\sqrt{\tau_{12}} \widetilde{N}_{3}, \\
& \widetilde{\Xi}_{16}(2)=\sqrt{\tau_{12}} \widetilde{N}_{2}, \\
& \widetilde{\Xi}_{66}=-\widetilde{R}_{5}, \\
& \widetilde{A}_{c}=A X E_{1}^{T}+B \bar{\Lambda} \widehat{K} E_{3}^{T}+B \bar{\Lambda} \widehat{K} E_{5}^{T}, \\
& \breve{A}_{c i}=B e_{i} \widehat{K}_{3}^{T}+B e_{i} \widehat{K} E_{5}^{T}
\end{aligned}
$$

and a desired controller gain matrix in (2) can be chosen as

$$
K=\widehat{K} X^{-1}
$$

\section{A Numerical Example}

In this section, a numerical example is used to illustrate the effectiveness and improvement of the proposed approach in this paper.

Consider a simplified model of an unstable batch reactor with parameters:

$$
\begin{aligned}
& A=\left[\begin{array}{cccc}
1.38 & -0.2077 & 6.715 & -5.676 \\
-0.5814 & -4.29 & 0 & 0.675 \\
1.067 & 4.273 & -6.654 & 5.893 \\
0.048 & 4.273 & 1.343 & -2.104
\end{array}\right], \\
& B=\left[\begin{array}{cc}
0 & 0 \\
5.679 & 0 \\
1.136 & -3.146 \\
1.136 & 0
\end{array}\right] .
\end{aligned}
$$

The eigenvalues of $A$ are 1.9910, 0.0635, -5.0566, and -8.6659; it is obvious that the unforced system (47) is unstable. The unstable system is controlled under a network which is described in Figure 1, and the event-triggered communication scheme (4) is introduced to optimize the performance of the NCSs. Considering the effect of the transmission delay, it is assumed that the lower bound of the network-induced delay $\tau_{1}=0.01 \mathrm{~s}$. The purpose of the design is to make this upper bound, $\tau_{2}$, as large as possible and the sampled data transmitted as low as possible so that the system can work under actuator failures conditions.

Two cases of probability distributions are considered.

Case 1. Actuator failures $\Lambda_{i}$ are Bernoulli distributed white sequences with parameters:

$$
\begin{aligned}
& E\left\{\Lambda_{i}=1\right\}=\bar{\Lambda}_{i} \geq 0, \\
& E\left\{\Lambda_{i}=0\right\}=1-\bar{\Lambda}_{i} .
\end{aligned}
$$

It is not difficult to verify that

$$
\beta_{i}^{2}=\bar{\Lambda}_{i}\left(1-\bar{\Lambda}_{i}\right)
$$

when $\delta=0$ and $\Omega_{1} \neq \Omega_{2}$. We conduct the simulation studies with different $\Lambda_{i}$. The maximal allowable delays $\tau_{2}$ and fault tolerant controller parameter $K$ from Corollary 11 and those in $[23,24]$ are listed in Table 1 . From Table 1 , it is clear that Corollary 11 provides the largest delay; that is, Corollary 11 is less conservative than all the other list results.

Case 2. Actuator failures $\Lambda_{i}$ are Gaussian distributed sequences with parameters.

Case 2.1. We have the following:

$$
\begin{aligned}
& \bar{\Lambda}_{1}=0.2, \\
& \bar{\Lambda}_{2}=0.3, \\
& \beta_{1}=0.5, \\
& \beta_{2}=0.6 .
\end{aligned}
$$

Case 2.2. We have the following:

$$
\begin{aligned}
& \bar{\Lambda}_{1}=0.2, \\
& \bar{\Lambda}_{2}=0.3, \\
& \beta_{1}=0.2, \\
& \beta_{2}=0.3
\end{aligned}
$$

when $\delta=0$ and $\Omega_{1} \neq \Omega_{2}$. Table 2 lists the maximal allowable delays $\tau_{2}$ and fault tolerant controller $K$ obtained from Corollary 11 and those in $[17,18]$. From Table 2, it can be concluded that our results are less conservative than the results from $[17,18]$.

In the following, under actuators failures $\Lambda_{i}$ in Case 2.2 with $\bar{\Lambda}_{1}=0.2, \bar{\Lambda}_{2}=0.3, \beta_{1}=0.2$, and $\beta_{2}=0.3$, we will demonstrate that the fault tolerant state-feedback controller also optimizes the performance of the NCSs, that is, considerable reduction of the network resource occupancy:

(1) When $\delta=0.1$ and $\Omega_{1} \neq \Omega_{2}$, applying Corollary 11, we can solve the maximum $\tau_{2}=0.23 \mathrm{~s}$, and the 
TABLE 1: The maximal allowable delays $\tau_{2}$ and fault tolerant controller $K$ for different $\Lambda_{i}$ in Case 1 .

\begin{tabular}{|c|c|c|c|c|c|c|}
\hline Case 1 & Maximal allowable delays & \multicolumn{5}{|c|}{$\bar{\Lambda}_{1}=0.4$ and $\bar{\Lambda}_{2}=0.5$} \\
\hline [17] & $\tau_{2}=0.11$ & $K=$ & {$\left[\begin{array}{l}0.4961 \\
3.7924\end{array}\right.$} & $\begin{array}{c}-0.1158 \\
0.7272\end{array}$ & $\begin{array}{l}0.3942 \\
2.9007\end{array}$ & $\left.\begin{array}{l}-0.3413 \\
-1.4987\end{array}\right]$ \\
\hline [18] & $\tau_{2}=0.12$ & $K=$ & {$\left[\begin{array}{c}-0.7941 \\
1.7764\end{array}\right.$} & $\begin{array}{c}-0.3913 \\
0.3258\end{array}$ & $\begin{array}{c}-0.6128 \\
1.2664\end{array}$ & $\begin{array}{r}0.0998 \\
-0.6207\end{array}$ \\
\hline Corollary 11 & $\tau_{2}=0.23$ & $K=$ & {$\left[\begin{array}{c}-1.5767 \\
1.7901\end{array}\right.$} & $\begin{array}{c}-0.4505 \\
0.4449\end{array}$ & $\begin{array}{r}-1.1426 \\
1.3089\end{array}$ & $\begin{array}{r}0.4351 \\
-0.5460\end{array}$ \\
\hline Case 1 & Maximal allowable delays & & $\bar{\Lambda}_{1}$ & $=0.2$ and & $\bar{\Lambda}_{2}=0.3$ & \\
\hline [17] & $\tau_{2}=0.08$ & $K=$ & {$\left[\begin{array}{l}0.9457 \\
6.5698\end{array}\right.$} & $\begin{array}{c}-0.2368 \\
1.2461\end{array}$ & $\begin{array}{l}0.7506 \\
5.0072\end{array}$ & $\left.\begin{array}{l}-0.6604 \\
-2.5903\end{array}\right]$ \\
\hline [18] & $\tau_{2}=0.10$ & $K=$ & {$\left[\begin{array}{c}-1.9127 \\
2.8661\end{array}\right.$} & $\begin{array}{c}-0.7303 \\
0.5948\end{array}$ & $\begin{array}{r}-1.4330 \\
2.0576\end{array}$ & $\begin{array}{r}0.3972 \\
-0.9473\end{array}$ \\
\hline Corollary 11 & $\tau_{2}=0.17$ & $K=$ & {$\left[\begin{array}{c}-3.2189 \\
3.4078\end{array}\right.$} & $\begin{array}{c}-0.9113 \\
0.8480\end{array}$ & $\begin{array}{r}-2.3315 \\
2.4877\end{array}$ & $\begin{array}{r}0.8898 \\
-1.0380\end{array}$ \\
\hline Case 1 & Maximal allowable delays & & $\bar{\Lambda}_{1}$ & $=0.1$ and & $\bar{\Lambda}_{2}=0.2$ & \\
\hline $\begin{array}{l}{[17]} \\
{[18]}\end{array}$ & $\begin{array}{c}\tau_{2}=- \\
\tau_{2}=0.08\end{array}$ & $K=$ & {$\left[\begin{array}{c}-2.9434 \\
4.8397\end{array}\right.$} & $\begin{array}{r}K=- \\
-1.2942 \\
0.9686\end{array}$ & $\begin{array}{l}-2.2716 \\
2.4877\end{array}$ & $\begin{array}{c}0.4601 \\
-1.0380\end{array}$ \\
\hline Corollary 11 & $\tau_{2}=0.12$ & $K=$ & {$\left[\begin{array}{c}-5.2739 \\
5.3644\end{array}\right.$} & $\begin{array}{r}-1.4717 \\
1.3453\end{array}$ & $\begin{array}{c}-3.8744 \\
3.9057\end{array}$ & $\begin{array}{r}1.4775 \\
-1.6241\end{array}$ \\
\hline
\end{tabular}

TABLE 2: The maximal allowable delays $\tau_{2}$ and fault tolerant controller $K$ for different $\Lambda_{i}$ in Case 2 .

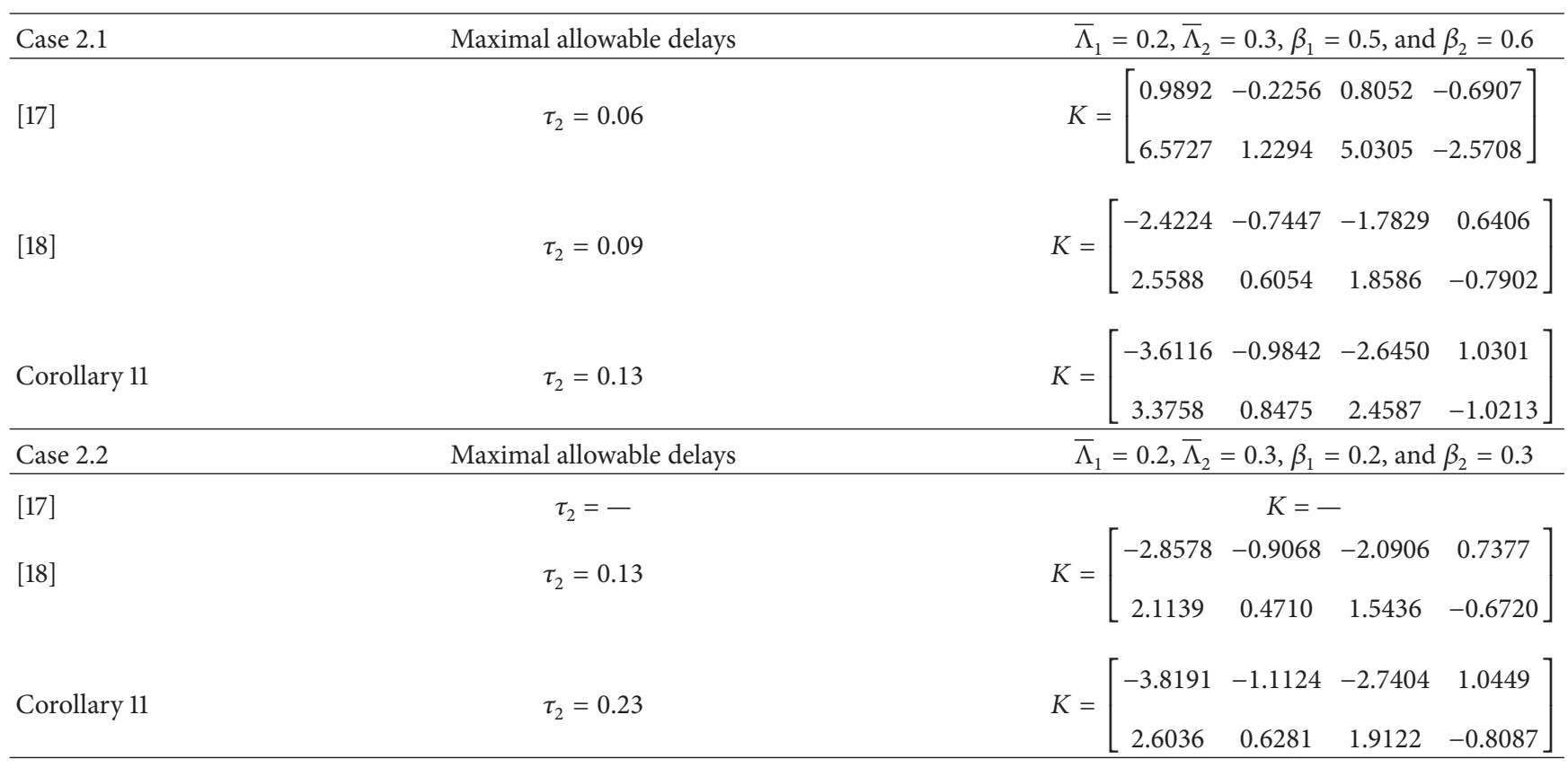


corresponding controller $K$ and the trigger parameter $\Omega_{1}$ and $\Omega_{2}$ are given by

$$
\begin{aligned}
K & =\left[\begin{array}{cccc}
-3.8157 & -1.1116 & -2.7398 & 1.0445 \\
2.5989 & 0.6263 & 1.9078 & -0.8073
\end{array}\right], \\
\Omega_{1} & =\left[\begin{array}{cccc}
0.0558 & 0.0173 & 0.0401 & -0.0150 \\
0.0173 & 0.0086 & 0.0113 & -0.0042 \\
0.0401 & 0.0113 & 0.0341 & -0.0157 \\
-0.0150 & -0.0042 & -0.0157 & 0.0087
\end{array}\right], \\
\Omega_{2} & =\left[\begin{array}{cccc}
171.9090 & 43.1360 & 125.6951 & -52.4983 \\
43.1360 & 10.8542 & 31.5284 & -13.1503 \\
125.6951 & 31.5284 & 91.9150 & -38.3995 \\
-52.4983 & -13.1503 & -38.3995 & 16.0563
\end{array}\right] .
\end{aligned}
$$

(2) When $\delta=0.1$ and $\Omega_{1}=\Omega_{2}$, applying Corollary 11, we can solve the maximum $\tau_{2}=0.15 \mathrm{~s}$, and the corresponding controller $K$ and the trigger parameter $\Omega$ are given by

$$
\begin{aligned}
& K=\left[\begin{array}{cccc}
-3.9951 & -1.3160 & -2.7469 & 0.9501 \\
3.0396 & 0.6144 & 2.2942 & -1.0344
\end{array}\right], \\
& \Omega=\left[\begin{array}{cccc}
3.3177 & 0.8513 & 2.4087 & -0.9850 \\
0.8513 & 0.2276 & 0.6132 & -0.2482 \\
2.0487 & 0.6132 & 1.7600 & -0.7259 \\
-0.9850 & -0.2482 & -0.7259 & 0.3034
\end{array}\right] .
\end{aligned}
$$

Remark 12. From Tables 1 and 2, it can be concluded that when $\delta=0$, that is, in the case of time-triggered communication scheme, our results are less conservative than existing ones. Moreover, when $\delta=0.1$, the maximal allowable delay $\tau_{2}$ obtained from Corollary 11 under condition $\Omega_{1} \neq \Omega_{2}\left(\tau_{2}=\right.$ $0.23 \mathrm{~s})$ is bigger than the case of $\Omega_{1}=\Omega_{2}\left(\tau_{2}=0.15 \mathrm{~s}\right)$; that is, two different weighting matrices $\Omega_{1}$ and $\Omega_{2}$ are introduced in (4) which guaranteed less conservative results.

Select the sampling period $h=0.10 \mathrm{~s}$. According to (7), we can see that the allowable maximum transmission delay can be $0.05 \mathrm{~s}$. The state response of system (47) with controller $K$ in (53) and $\Omega$ in (54) is shown in Figure 2. Figure 3 plots the release instants and release interval.

From Figures 2 and 3, we can see that system (47) remains asymptotically stable under the feedback controller $K$ in (53) and trigger matrix $\Omega$ in (54); the simulation results for $t \in[0,30]$ show that only 58 sampled signals need to be transmitted to the controller through the transmitter, which takes $19.33 \%$ of the whole sample signals. Thus, the fault tolerant state-feedback controller presented in this paper also optimizes the performance of the NCSs by reducing the networked load.

Remark 13. The simulation results show that the amount of transferred data is reduced by introducing the event-triggering communication scheme. In addition, the maximum

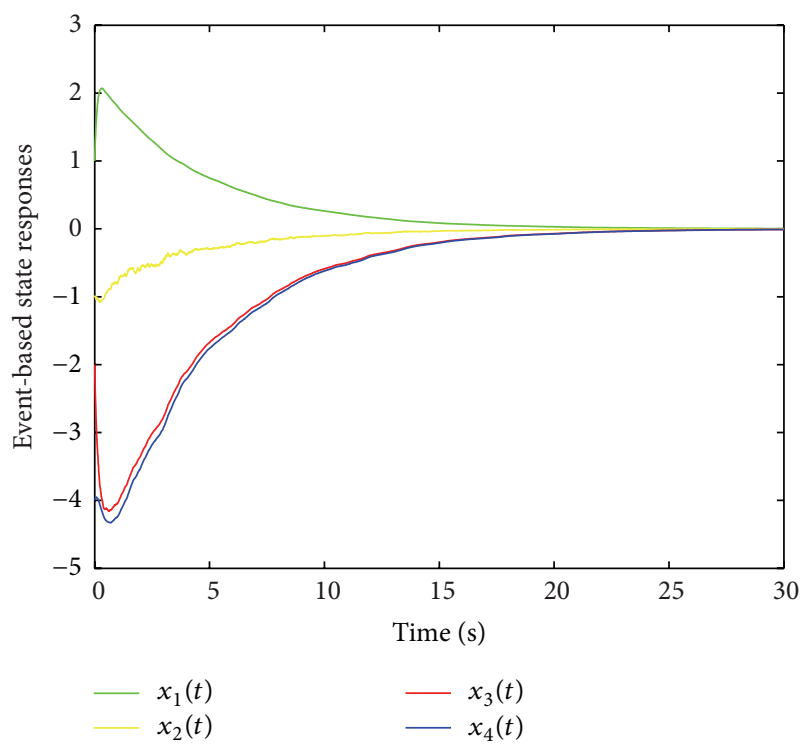

Figure 2: The state responses of system (47) under the feedback controller $K$ in (53) and trigger matrix $\Omega$ in (54).

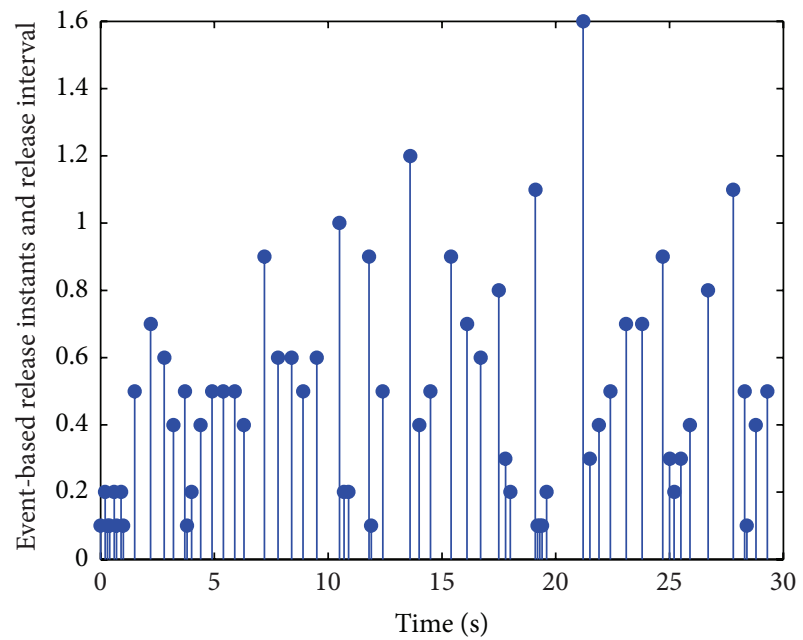

FIGURE 3: The release instants and release interval under the feedback controller $K$ in (53) and trigger matrix $\Omega$ in (54).

value, $\tau_{2}$, in this paper is bigger than the value, $\tau_{2}$, in $[17,18]$. Therefore, the proposed controller design method in the paper not only is less conservative but also has less transferred data than $[17,18]$.

\section{Conclusion}

The fault tolerant state-feedback controller design problem for stochastic NCSs has been investigated in the paper. Improved state-feedback controller design method with less conservation and less sampled data transmission has been proposed by employing event-triggered communication scheme, the delay-decomposition technique, and convex combination approach. A practical numerical example for the stabilization of batch reactor has been given to demonstrate 
that the theory represented in this paper is less conservative than existing ones, and introducing event-triggered communication scheme can optimize the performance of stochastic NCSs. Therefore, the proposed controller design method in the paper is an improvement over existing ones.

\section{Competing Interests}

The authors declare that there is no conflict of interests regarding the publication of this paper.

\section{Acknowledgments}

This work was supported in part by the National Natural Science Funds for Young Scholar of China under Grant 61304046, Natural Science Foundation of Heilongjiang Province of China (Grant no. QC 2015064), and Scientific Research Foundation for the Returned Overseas Chinese Scholars, Heilongjiang Province of China (Grant no. LC 2016027).

\section{References}

[1] R.-A. Gupta and M.-Y. Chow, "Networked control system: overview and research trends," IEEE Transactions on Industrial Electronics, vol. 57, no. 7, pp. 2527-2535, 2010.

[2] L. Zhang, H. Gao, and O. Kaynak, "Network-induced constraints in networked control systems-a survey," IEEE Transactions on Industrial Informatics, vol. 9, no. 1, pp. 403-416, 2013.

[3] F. Li, P. Shi, L. Wu, M. V. Basin, and C.-C. Lim, "Quantized control design for cognitive radio networks modeled as nonlinear semi-Markovian jump systems," IEEE Transactions on Industrial Electronics, vol. 62, no. 4, pp. 2330-2340, 2015.

[4] Y. Shi and B. Yu, "Robust mixed $H_{2} / H_{\infty}$ control of networked control systems with random time delays in both forward and backward communication links," Automatica, vol. 47, no. 4, pp. 754-760, 2011.

[5] R. Yang, P. Shi, and G.-P. Liu, "Filtering for discrete-time networked nonlinear systems with mixed random delays and packet dropouts," IEEE Transactions on Automatic Control, vol. 56, no. 11, pp. 2655-2660, 2011.

[6] E. Tian and D. Yue, "Reliable $H_{\infty}$ filter design for T-S fuzzy model-based networked control systems with random sensor failure," International Journal of Robust and Nonlinear Control, vol. 23, no. 1, pp. 15-32, 2013.

[7] S. H. Kim, " $\mathscr{H}_{\infty}$ output-feedback tracking control for networked control systems," Mathematical Problems in Engineering, vol. 2015, Article ID 724389, 10 pages, 2015.

[8] J. Mazo and P. Tabuada, "Decentralized event-triggered control over wireless sensor/actuator networks," IEEE Transactions on Automatic Control, vol. 56, no. 10, pp. 2456-2461, 2011.

[9] Q. Liu, Z. Wang, X. He, and D. Zhou, "A survey of eventbased strategies on control and estimation," Systems Science and Control Engineering, vol. 2, no. 1, pp. 90-97, 2014.

[10] C. Peng and T. C. Yang, "Event-triggered communication and $H_{\infty}$ control co-design for networked control systems," Automatica, vol. 49, no. 5, pp. 1326-1332, 2013.

[11] H. Yan, S. Yan, H. Zhang, and H. Shi, "Event-triggered $H_{\infty}$ control for networked control systems with time-varying delay,"
Mathematical Problems in Engineering, vol. 2014, Article ID 874823, 7 pages, 2014.

[12] S. Hu, X. Yin, Y. Zhang, and E. G. Tian, "Event-triggered guaranteed cost control for uncertain discrete-time networked control systems with time-varying transmission delays," IET Control Theory and Applications, vol. 6, no. 18, pp. 2793-2804, 2012.

[13] X.-M. Zhang and Q.-L. Han, "Event-triggered dynamic output feedback control for networked control systems," IET Control Theory \& Applications, vol. 8, no. 4, pp. 226-234, 2014.

[14] D. Zhang, Q.-L. Han, and X. Jia, "Network-based output tracking control for T-S fuzzy systems using an event-triggered communication scheme," Fuzzy Sets and Systems, vol. 273, pp. 2648, 2015.

[15] H. Wang, P. Shi, and J. Zhang, "Event-triggered fuzzy filtering for a class of nonlinear networked control systems," Signal Processing, vol. 113, pp. 159-168, 2015.

[16] S. Hu, D. Yue, X. Xie, and Z. Du, "Event-triggered $H_{\infty}$ stabilization for networked stochastic systems with multiplicative noise and network-induced delays," Information Sciences, vol. 299, pp. 178-197, 2015.

[17] C. Peng, T. C. Yang, and E. G. Tian, "Brief paper: robust faulttolerant control of networked control systems with stochastic actuator failure," IET Control Theory \& Applications, vol. 4, no. 12, pp. 3003-3011, 2010.

[18] S. Wang, J. Feng, and H. Zhang, "Robust fault tolerant control for a class of networked control systems with state delay and stochastic actuator failures," International Journal of Adaptive Control and Signal Processing, vol. 28, no. 9, pp. 798-811, 2014.

[19] Y. Zhai, R. Yan, H. Liu, and J. Liu, "Event-triggered reliable control in networked control systems with probabilistic actuator faults," Mathematical Problems in Engineering, vol. 2013, Article ID 131942, 9 pages, 2013.

[20] J. Liu, "Event-based reliable $H_{\infty}$ control for networked control system with probabilistic actuator faults," Chinese Journal of Electronics, vol. 22, no. 4, pp. 843-848, 2013.

[21] J. Liu and D. Yue, "Event-triggering in networked systems with probabilistic sensor and actuator faults," Information Sciences, vol. 240, pp. 145-160, 2013.

[22] X. Su, P. Shi, L. Wu, and Y. Song, "Fault detection filtering for nonlinear switched stochastic systems," IEEE Transactions on Automatic Control, vol. 61, no. 5, pp. 1310-1315, 2015.

[23] N. Touijer and S. Kamoun, "Design of robust self-tuning control schemes for stochastic systems described by input-output mathematical models," International Journal of Innovative Computing, Information and Control, vol. 11, no. 3, pp. 1101-1112, 2015.

[24] X. Su, L. Wu, P. Shi, and C. L. Philip Chen, "Model approximation for fuzzy switched systems with stochastic perturbation," IEEE Transactions on Fuzzy Systems, vol. 23, no. 5, pp. 14581473, 2015.

[25] F. Li, L. Wu, P. Shi, and C.-C. Lim, "State estimation and sliding mode control for semi-Markovian jump systems with mismatched uncertainties," Automatica, vol. 51, pp. 385-393, 2015.

[26] F. Li, P. Shi, L. Wu, and X. Zhang, "Fuzzy-model-based Dstability and nonfragile control for discrete-time descriptor systems with multiple delays," IEEE Transactions on Fuzzy Systems, vol. 22, no. 4, pp. 1019-1025, 2014.

[27] B. Song, J. H. Park, Z.-G. Wu, and X. Li, "New results on delay-dependent stability analysis and stabilization for stochastic time-delay systems," International Journal of Robust and Nonlinear Control, vol. 24, no. 16, pp. 2546-2559, 2014. 
[28] L. Huang, X. Xie, Y. Zhao, and Z. Hu, "Disturbance attenuation observer design for fuzzy time-delay system with fault," Journal of Intelligent and Fuzzy Systems, vol. 30, no. 2, pp. 811-819, 2016.

[29] D. Yue, E. Tian, Y. Zhang, and C. Peng, "Delay-distributiondependent stability and stabilization of T-S fuzzy systems with probabilistic interval delay," IEEE Transactions on Systems, Man, and Cybernetics, Part B: Cybernetics, vol. 39, no. 2, pp. 503-516, 2009.

[30] S. Boyd, L. Ghaoui, E. Feron, and V. Balakrishnan, Linear Matrix Inequalities in System and Control Theory, Society for Industrial and Applied Mathematics Press (SIAM), Philadelphia, Pa, USA, 1994. 

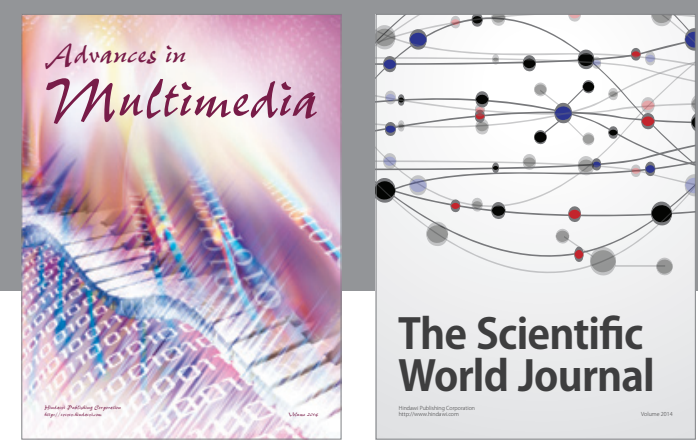

The Scientific World Journal
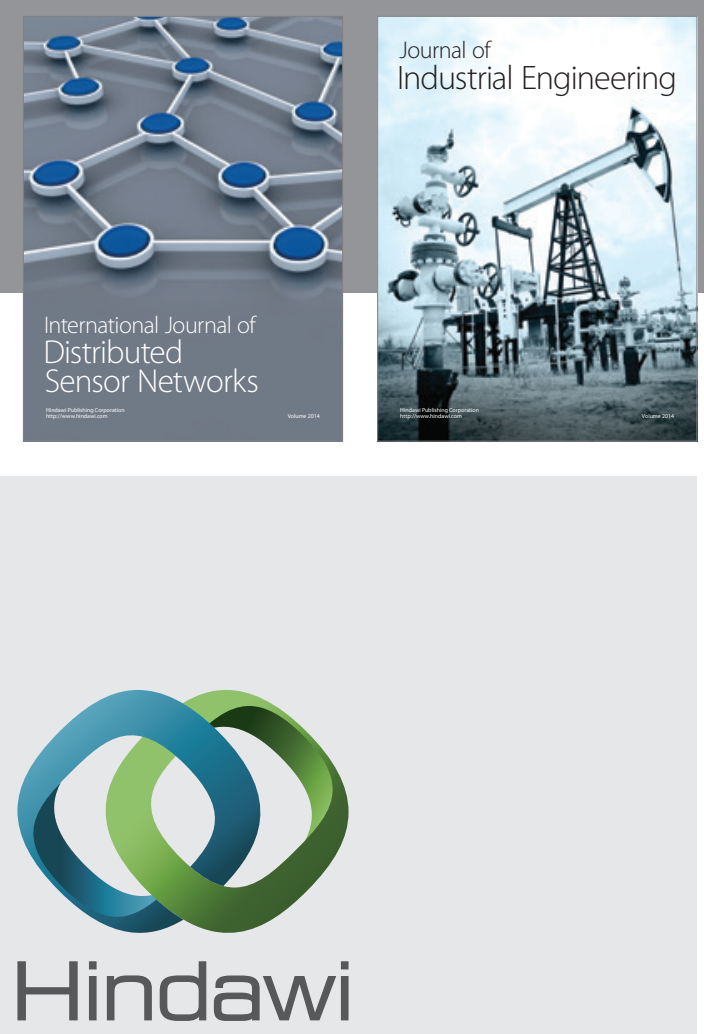

Submit your manuscripts at

http://www.hindawi.com

\section{Computer Networks} and Communications
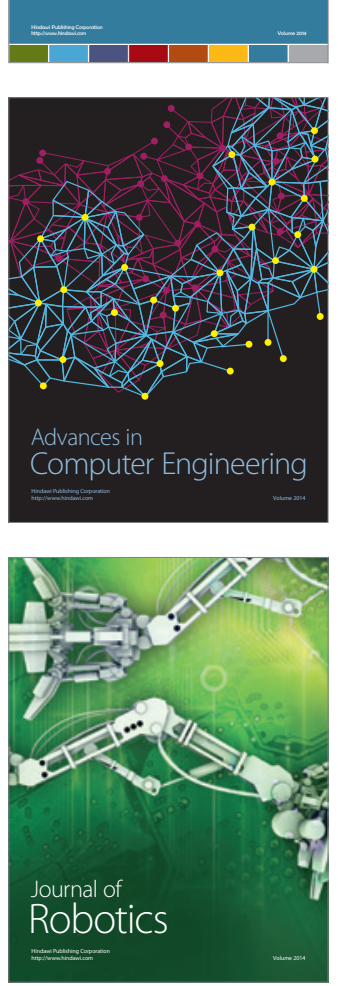
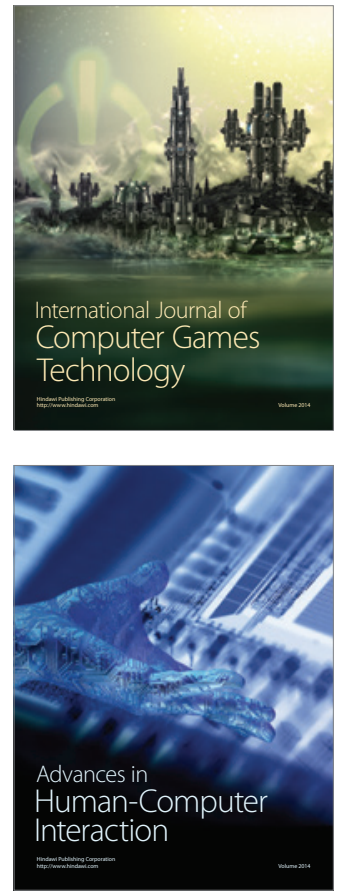
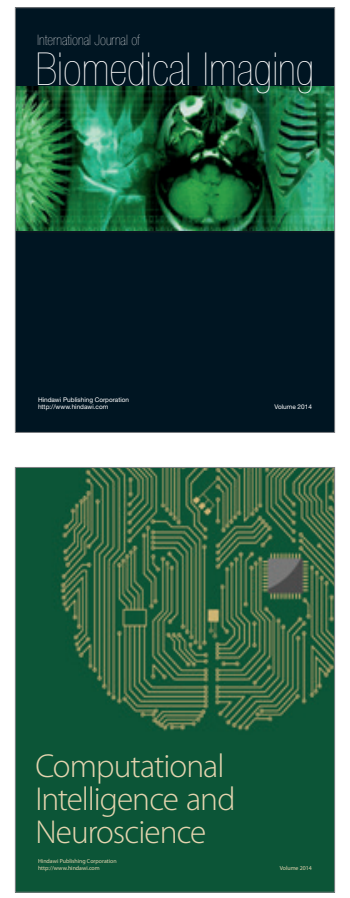
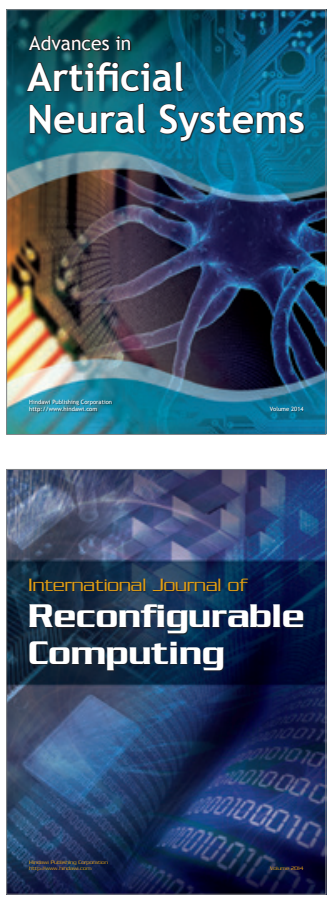
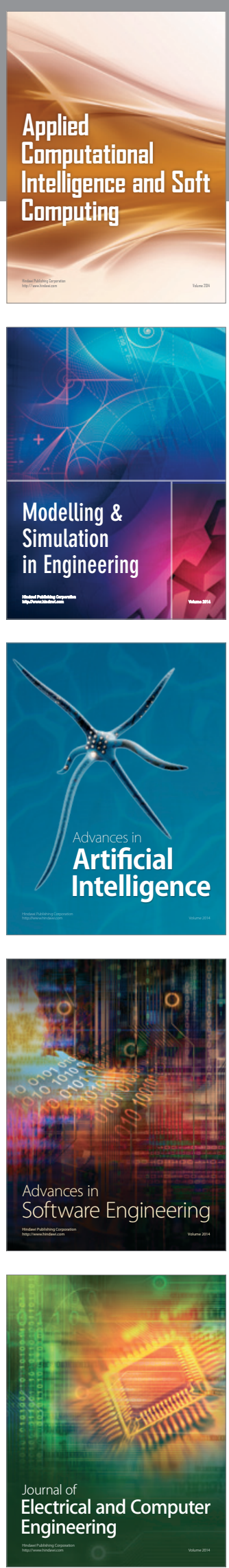